\title{
Position Control of the Surface Motor Based on Artificial Neural Network
}

\author{
Abdulhasan F. Abdulhasan ${ }^{1}$, Rabee' H. Thejel ${ }^{2}$, Diyah K. Shary ${ }^{3}$ \\ \{abd.allami@stu.edu.iq, rabee_alabbasi@stu.edu.iq,diyahpower@stu.edu.iq\} \\ ${ }^{1-3}$ Department of Electrical Power Techniques Engineering, Southern Technical University, Iraq
}

\begin{abstract}
The step movement of the switched reluctance planar motor (SRPM) has high oscillation, due to the nonlinear magnetic circuit behavior and high force ripples. Therefore, traditional linear controllers are insufficient in SRPM control, thus a closed-loop motor position control system based on Neural-PID controller is designed to obtain the optimum characteristics of operation for these motors.
\end{abstract}

Keywords: switched reluctance planar motor, neural network controller, PID controller, position control of the planar motor.

\section{Introduction}

The planar motor also named Surface Motor (SFM) uses electromagnetic force to achieve a high precision movement without friction [1]. The two-dimensional movement of the planar motor is accurate and fast and used in many laboratory and industrial applications. These motors offer certain benefits over the rotating motor due to the absence of the rotary-to-linear transformation mechanisms in their XY movements [2]. Consequently, the direct-drive surface motors can be expected that they will replace using the traditional XY movement that achieves twodimensional movement by using a rotary machine with mechanical transformation or using two linear motors fixed one on the other [3]. The surface motor is the switched reluctance planar motor (SRPM) that consists of the mover (armature) part and the platen (stator) part [4]. It is a robust, low-cost, and simple motor. With the requirements of the simple converter and control, the SRPM is gaining much attention in the drive applications in the industry [5]. Due to the nonlinear magnetic circuit and high force ripples, the step position of SRPM has high oscillations and overshoots during the step movement, and it is difficult to reduce the oscillations and achieve the precision position without using a good control method.

Many control strategies were applied for SRPM to realize position control. In [5], the adaptive control based on the pole-placement method was designed and implemented with parameter setting online for SRPM. The performance of this controller has good position control over the PID controller in static, dynamic, and robustness to disturbances. In [6], improved the SRPM with an optimized structural design to reduce the electromagnetic force ripple. The results prove the optimized SRPM has a higher drive force and the optimization design of the SRPM is effective in reducing the ripple of the electromagnetic force. In [7], designed a DSP-based sliding mode controller of the SRPM for accurate locating applications. The position errors for $\mathrm{X}$ and $\mathrm{Y}$ directions are 0.016 and $0.029 \mathrm{~mm}$, respectively. In [8], a predictive position control (PPC) of the PSRMs for XY movement systems is given. By reducing the cost function based on the PPC, the optimal control sequence is derived. The methods above have been applied to SRPMs drive, but there is no oscillation elimination for the position response of the SRPM.

In this paper, the Neural-PID controller is proposed and designed for the SRPM drive circuit to reduce the position oscillation.

\section{The Modeling of SRPM}

The modular of the switched reluctance planar motor consists of six phases of switched reluctance motor (SRM), three for the movement in $X$-direction $\left(X_{1}, X_{2}, X_{3}\right)$ and three for movement in Y-direction $\left(Y_{1}, Y_{2}, Y_{3}\right)$ [2]. The mathematical model of the SRPM for one phase in the X-direction that is given in [9], [10] can be implemented using Matlab/Simulink as is shown in Fig 1. Subsystem 1 and subsystem 2 of Fig 1 are shown in Figs 2 and 3, respectively. All other phases of SRPM can be implemented in the same way. 


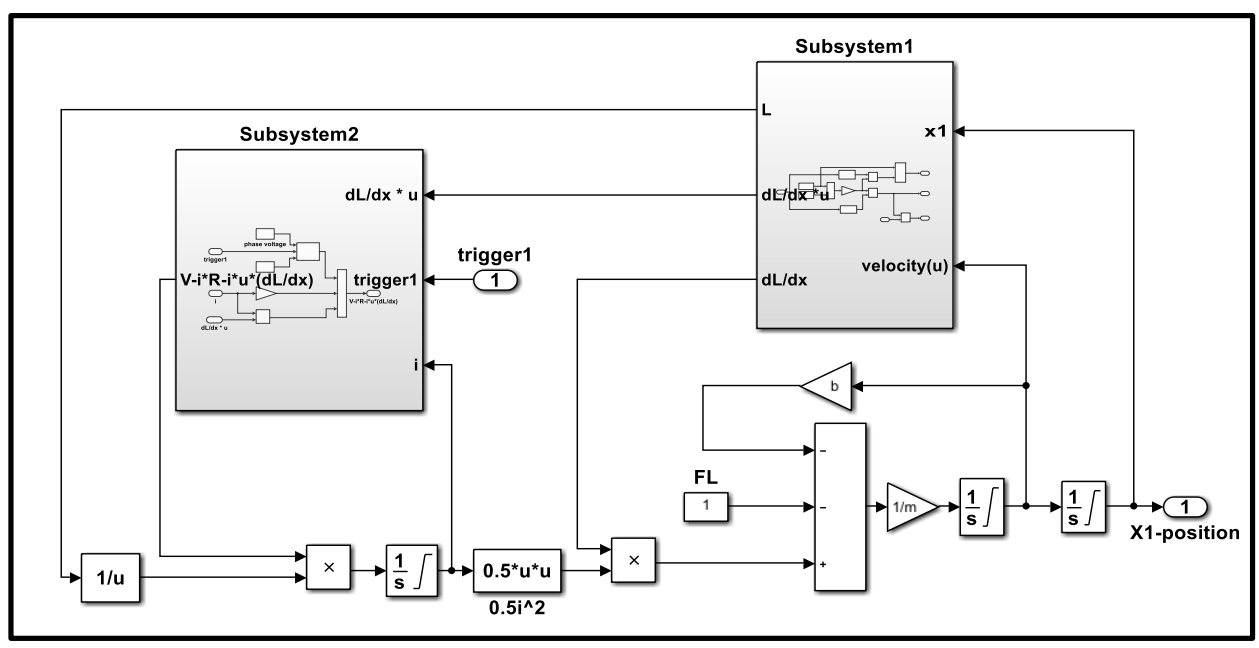

Fig 1. Implemented Simulink model of one phase of SRPM in the X-direction.

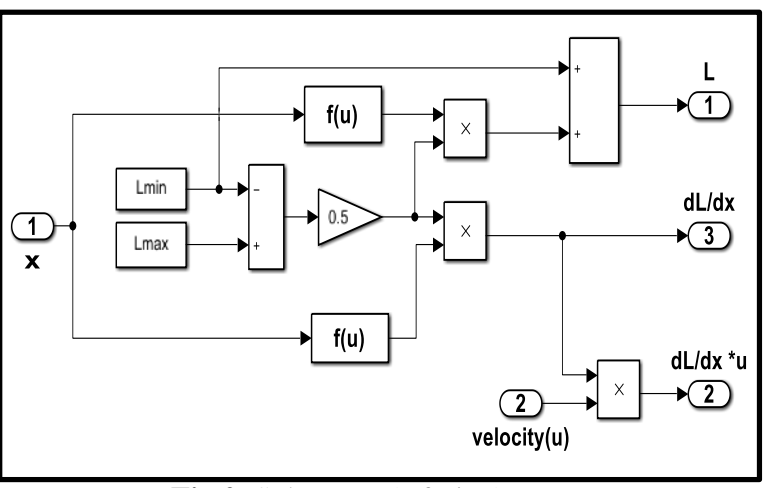

Fig 2. Subsystem1 of Fig.1.

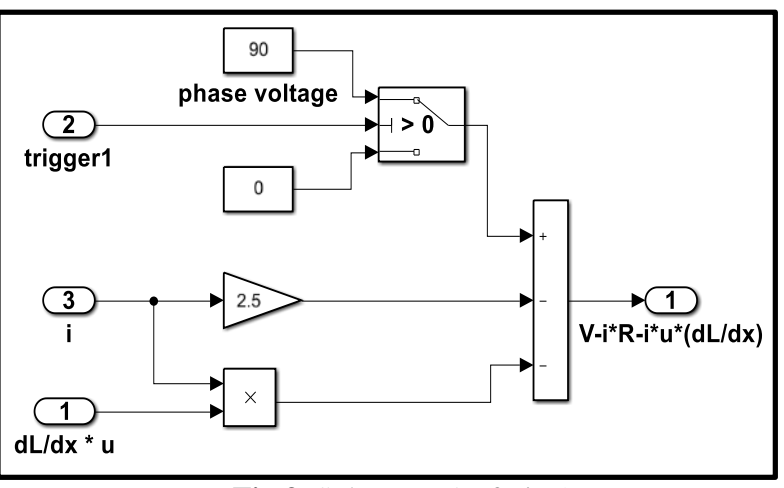

Fig 3. Subsystem2 of Fig.1

\section{The SRPM Controller}

The switched reluctance planar motor is a non-linear system. Therefore, it is not easy to obtain a good result for SRPM using the traditional linear control methods. Thus, the performance of the traditional PID controller has been insufficient due to the nonlinear structure of the surface motor. In this work, the performance and accuracy requirements of the SRPM position control system are done by integrating a neural network with a PID controller. The complete controller type position control-based surface motor drive system is proposed as shown in Fig 4. Where, $\mathrm{X}_{\mathrm{off}}$ is the off-position of the mover in $(\mathrm{m}), \mathrm{X}_{\text {ref }}$ is the reference position of the motor in $(\mathrm{m}), \mathrm{X}$ is the actual (mover) position in $(\mathrm{m})$, and $\mathrm{e}(\mathrm{t})$ is the error between the reference and actual positions.

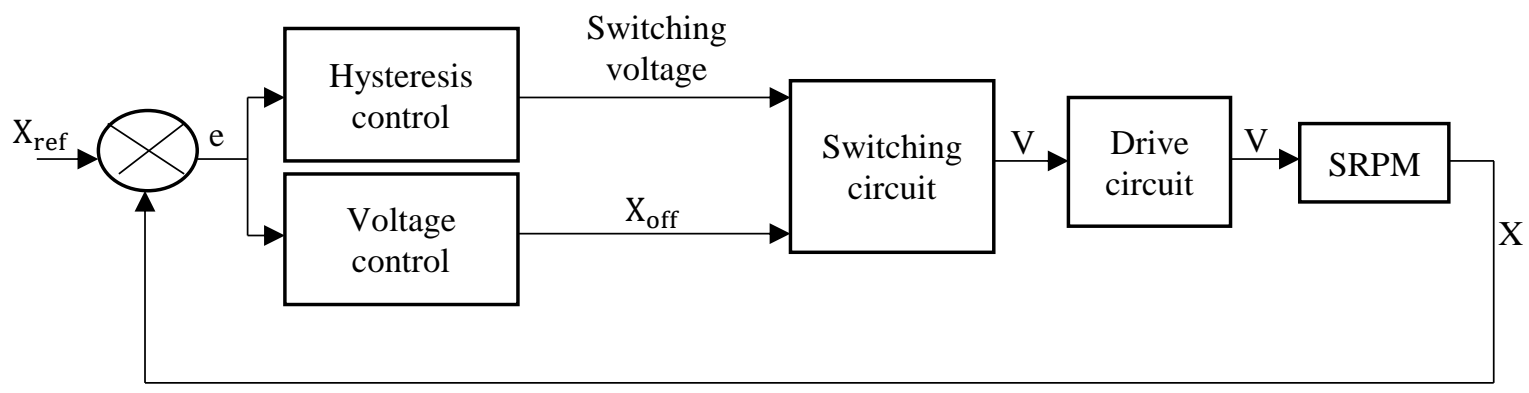

Fig 4. The configuration of SRPM with the proposed controller. 
In this work, the controller contains two-controller blocks, the position- $\mathrm{X}_{\text {off }}$ controller (voltage control) and position- $\mathrm{I}_{\text {ref }}$ controller (hysteresis control). These controllers are supplied to a switching circuit as shown in Fig 5. A Neural Network (NN) controller is used to tune the parameters of PID controllers of hysteresis and voltage control.

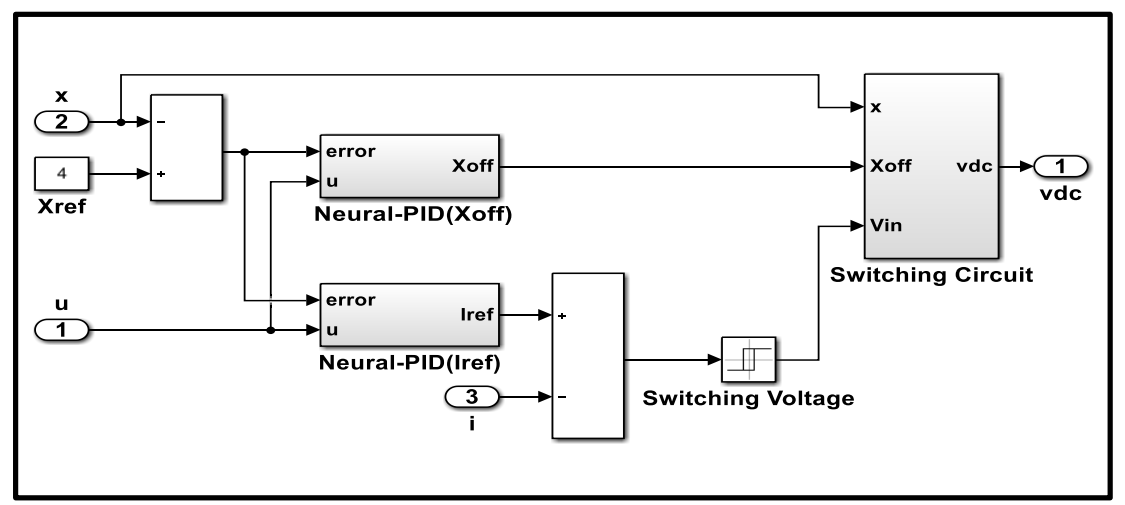

Fig 5. Position- $\mathrm{X}_{\text {off }}$ and position- $\mathrm{I}_{\text {ref }}$ controllers with switching circuit.

\subsection{Position- Iref controller (Hysteresis Control)}

In this control method, the current is adjusted between two current levels equal to $\mathrm{I}_{\text {ref }} \pm \Delta \mathrm{i}$, where, $\Delta \mathrm{i}$ is the hysteresis band (chopping width) and $\mathrm{I}_{\text {ref }}$ is the reference current. The reference position $\mathrm{X}_{\mathrm{ref}}$ is compared with the mover position $\mathrm{X}$ to give the position error. The position error signal is handled by the PID position controller to produce the reference current as shown in Fig 6. The errors estimate by comparing the reference current with the actuator current and it is used to limit the switching voltage that is supplied to the switching circuit as shown in Fig 5 .

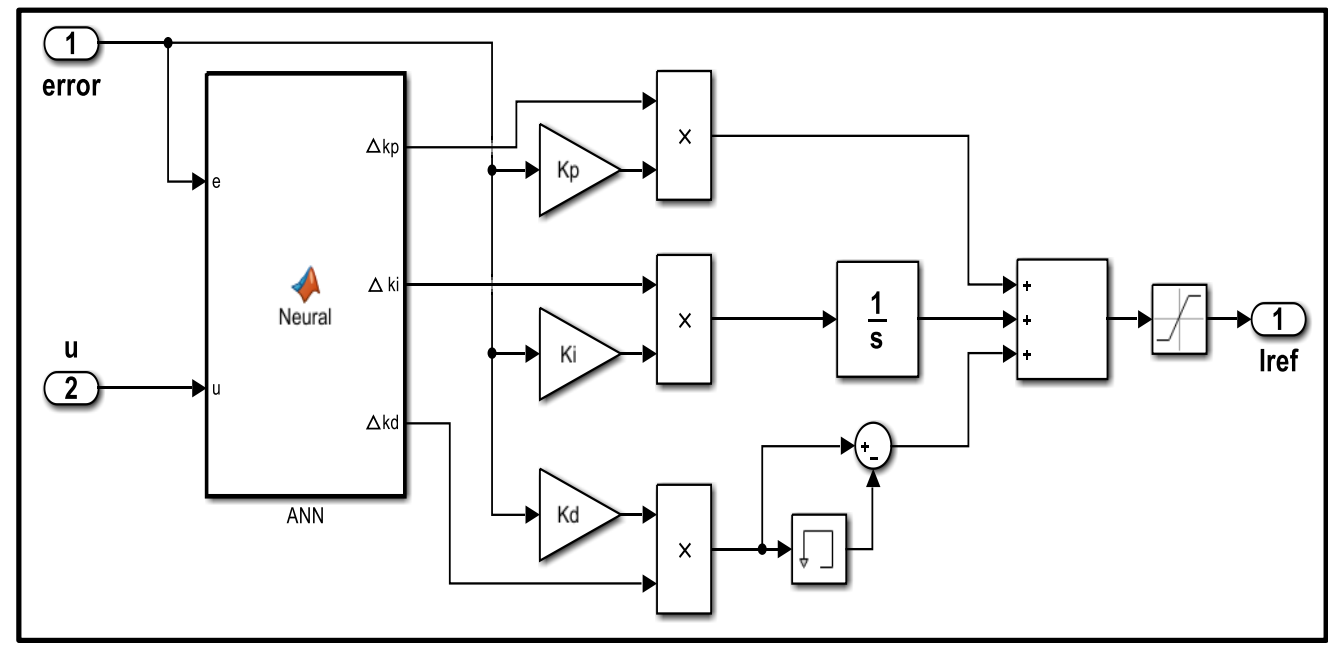

Fig 6. Implemented Simulink model of position- $\mathrm{I}_{\text {ref }}$ controller.

The switching logic of the current chopping controller is summarized as [11]:

if $\left(I_{\text {ref }}-i\right) \geq \Delta$ i then $V$ in $=V_{d c}$

if $\left(\mathrm{I}_{\text {ref }}-\mathrm{i}\right) \leq-\Delta \mathrm{i}$ then $\mathrm{Vin}=-\mathrm{V}_{\mathrm{dc}}$

else $\mathrm{e}=0$

$\Delta \mathrm{i}$ is chosen to be 0.1 and $\mathrm{V}_{\mathrm{dc}}$ is 90 Volt.

where, i, and Vin are the phase current (actuator current), and the switching voltage, respectively.

The parameters of the PID controller are tuned online by the NN. In this paper two input neurons (error (e), velocity (u)), five hidden neurons, and three output neurons (the change in parameters gains of PID controller) have been chosen as shown in Fig 7. 


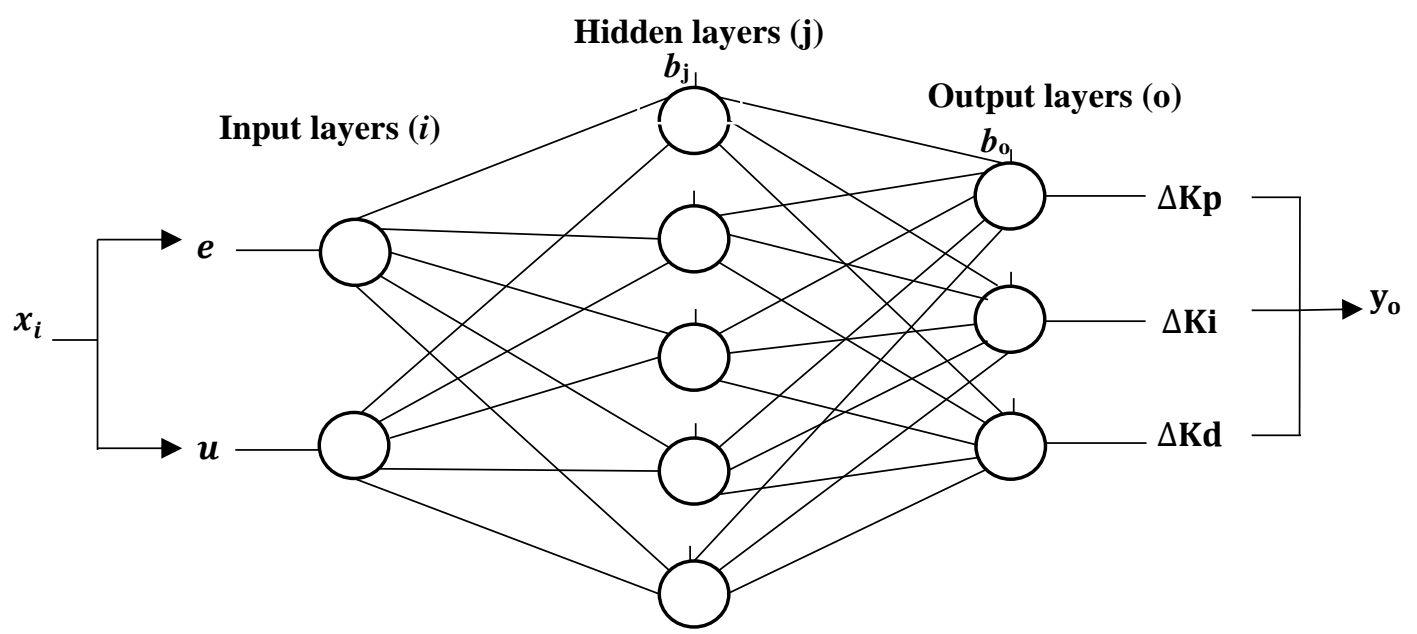

Where,

Fig 7. Structure of NN controller.

$x_{i}=\left[\begin{array}{l}e \\ u\end{array}\right]$ and $\mathrm{y}_{\mathrm{o}}=\left[\begin{array}{c}\Delta \mathrm{Kp} \\ \Delta \mathrm{Ki} \\ \Delta \mathrm{Kd}\end{array}\right]$

The mathematical equation that describes the neural network can be calculated by the following equations [12]:

$S_{j}^{k}=\sum_{i=1}^{N} w_{j i}^{k} \cdot x_{i}^{k}+b_{j}^{k}$

$y_{j}^{k}=\frac{1}{1+e^{-S_{j}^{k}}}$

$S_{o}^{k}=\sum_{j=1}^{n} w_{o j}^{k} \cdot y_{j}^{k}+b_{o}^{k}$

$y_{o}^{k}=\frac{1}{1+e^{-S_{O}^{k}}}$

where, $y_{j}^{k}, y_{o}^{k}, W_{j i}, W_{o j}, b_{j}^{k}, b_{o}^{k}, k, K$ are the output of hidden layers, the output of NN, the weights between the hidden and the input neurons, the weights between the output neuron and the hidden neurons, the bias of hidden layers, the bias of output layers, the present training set and $k=1: K$, and the total no. of the training sets, respectively.

In this work, the backpropagation training algorithm was used to train the weights of $\mathrm{NN}$. The weights are altered in the backward direction by the delta rules equations of the backpropagation algorithm as follow:

$\partial=y_{o}^{k} \cdot\left(1-y_{o}^{k}\right) \cdot\left(d^{k}-y_{o}^{k}\right)$

$\partial_{h}=y_{j}^{k} *\left(1-y_{j}^{k}\right) * w_{o j}^{k} * \partial$

$w_{o j}^{k+1}=w_{o j}^{k}+\partial^{*} \eta^{*} y_{j}^{k}$

$b_{o}^{k+1}=b_{o}^{k}+\partial^{*} \eta$

$w_{j i}^{k+1}=w_{j i}^{k}+\partial_{h} * \eta^{*} x_{i}^{k}$

$b_{j}^{k+1}=b_{j}^{k}+\partial_{h} * \eta$

where $\eta, e_{\max }, \partial, \partial_{h}$ are the learning ratio which is chosen to be 0.5 , the maximum error, the delta rule of the output neurons, and the delta rule of the hidden neurons, respectively.

The flow chart of the algorithm architecture of the backpropagation learning program is shown in Fig 8 . The input data $\left(x_{i}\right)$ is represented as a vector with rows equal to $(\mathrm{K})$ and its columns equal to the number of input neurons. The desired output data (d) is expressed as a column vector with $\mathrm{K}$ rows. 


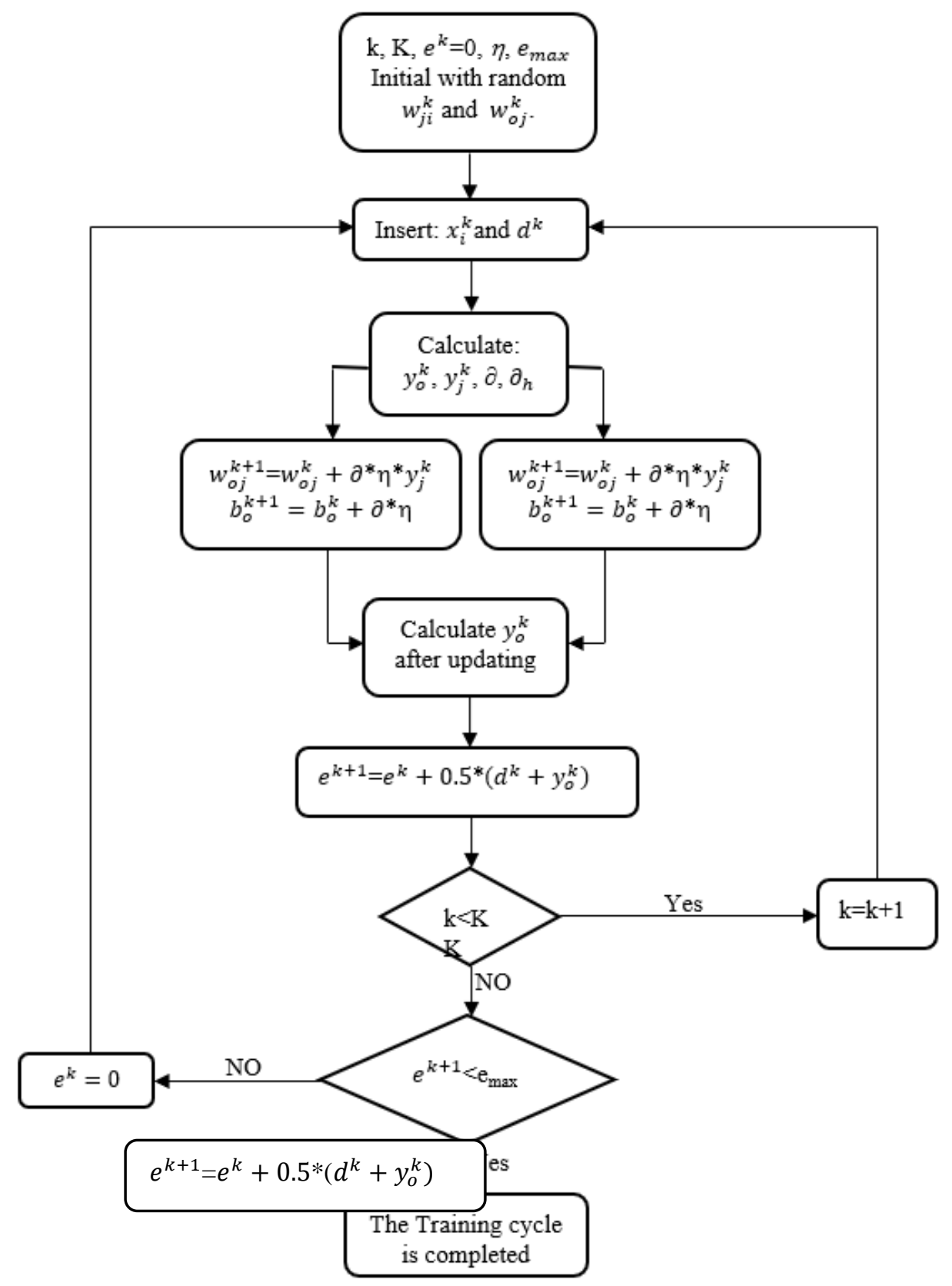

Fig 8. Flow chart of the backpropagation training.

The neural network parameters are:

1-The weights of the hidden layers:

$$
w_{j i}=\left[\begin{array}{cc}
0.7950 & 0.1905 \\
0.8574 & 0.2478 \\
-1.0578 & 0.4141 \\
-0.9118 & 0.0548 \\
0.3957 & 0.9090
\end{array}\right], h_{j}=\left[\begin{array}{c}
2.0662 \\
1.8304 \\
-0.1093 \\
0.8324 \\
1.9222
\end{array}\right]
$$


2-The weights of the output layers:

$w_{o j}=\left[\begin{array}{ccccc}-1.7848 & -1.9092 & -0.1539 & -1.2367 & -1.6983 \\ -2.0520 & -0.8829 & 0.0322 & -0.6682 & -1.3627 \\ -1.8352 & -1.5920 & -0.0895 & -1.5324 & -1.8329\end{array}\right], h_{o}=\left[\begin{array}{c}12.0682 \\ 9.4067 \\ 6.2037\end{array}\right]$

\subsection{Position- $X_{\text {off }}$ controller (voltage control)}

This control occurs by applying a voltage on phases of SRPM from the turn-on position $\left(\mathrm{X}_{\mathrm{on}}\right)$ period to a turnoff position $\left(\mathrm{X}_{\text {off }}\right)$ period. After that, the applied voltage is reversed completely until a certain demagnetizing position $\left(\mathrm{X}_{\mathrm{q}}\right)$, which allows the return of the magnetic flux towards zero. The mover position at which the stator current becomes zero is called the demagnetizing position $\left(\mathrm{X}_{\mathrm{q}}\right)$. The correct decision of the period-off position and the value of current determine the performance of SRM. In this controller, the Neural-PID controller is used to select the appropriate off-position of the motor as is shown in Fig 9. The output of this controller is supplied to a switching circuit as shown in Fig 5. The NN is used to tune the parameters of the PID controller. In this controller, the structure and learning algorithms of $\mathrm{NN}$ are the same that is used in position- $\mathrm{I}_{\text {ref }}$ controller.

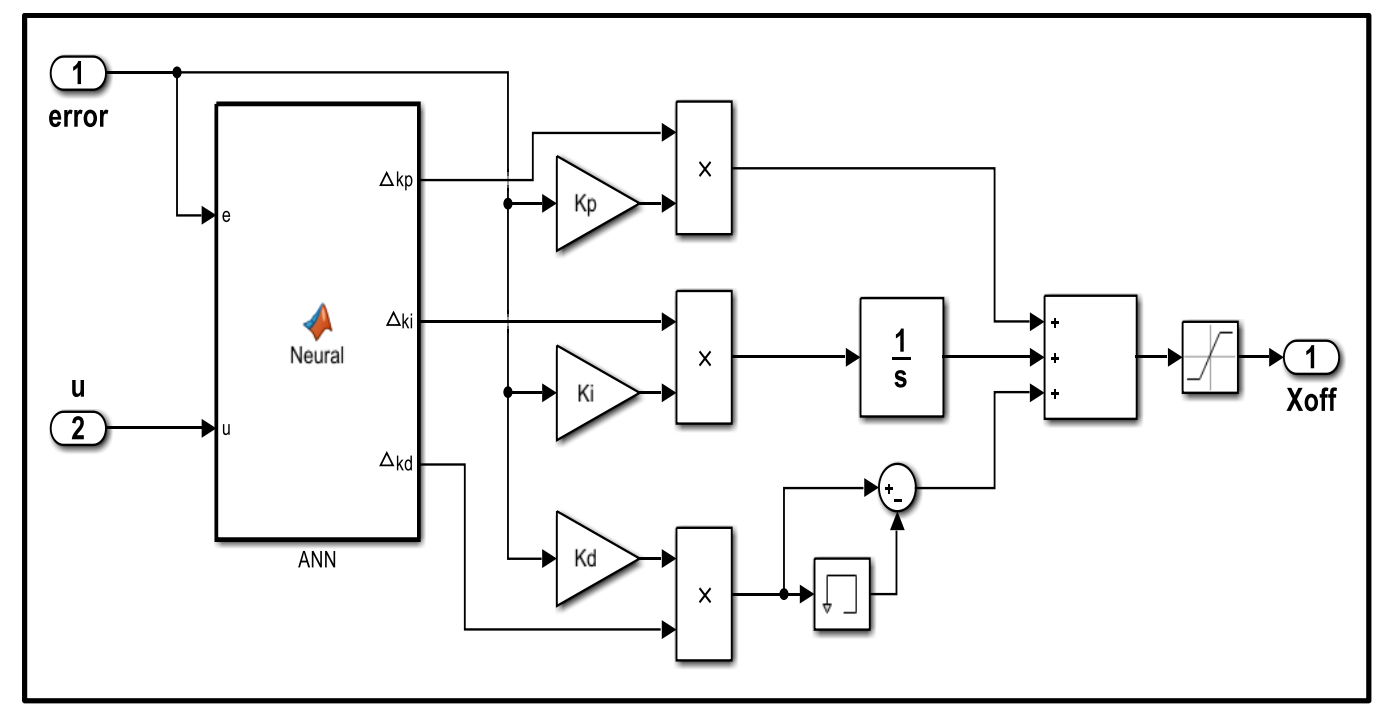

Fig 9. Implemented Simulink model of position- $\mathrm{X}_{\text {off }}$ controller.

The neural network parameters are:

1-The weights of hidden layers:

$w_{j i}=\left[\begin{array}{cc}0.5265 & -23.0829 \\ 1.5274 & 19.9201 \\ 13.9150 & -13.9376 \\ 1.4507 & -18.8408 \\ 1.5747 & -1.0786\end{array}\right], h_{j}=\left[\begin{array}{c}6.7429 \\ -1.1446 \\ -0.2194 \\ 4.4439 \\ -2.5557\end{array}\right]$

2-The weights of output layers:

$w_{o j}=\left[\begin{array}{ccccc}2.4602 & -7.0001 & 39.7432 & 2.8495 & 15.5845 \\ 3.0966 & 2.9797 & -8.8762 & -3.4552 & -4.7579 \\ 7.2849 & -3.0789 & 3.8074 & 2.1286 & 5.8654\end{array}\right], h_{o}=\left[\begin{array}{c}-19.2974 \\ 1.3311 \\ -3.9006\end{array}\right]$ 


\subsection{Switching Circuit}

The movement of the SFM is achieved by energizing and de-energizing the phase windings. The selection of conduction positions plays important role in the force ripple and the power of SRPM. However, it is hard to pick the proper conduction position [11]. In this work, the switching circuit is used to compare the mover position (X) for each phase with the turn-on position, turn-off position, and demagnetizing position to give the appropriate voltage supply for SRM as shown in Fig 10. Currently, the performance of SRPM is done by keeping the on position constant and changing the off position of SRPM. $X_{\text {on }}$ and $X_{q}$ values are chosen to be $0 \mathrm{~mm}$ and $3 \mathrm{~mm}$, respectively.

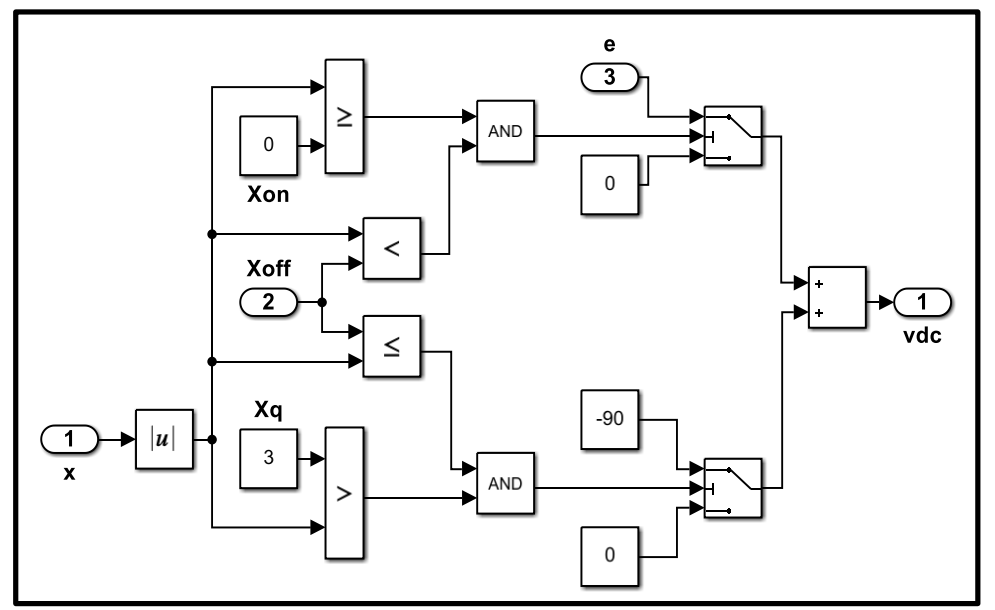

Fig 10. Switching circuit of the SRPM.

\section{Simulation Results}

The modeling with the control circuits of the SRPM was implemented using Matlab/Simulink software. The parameters of one phase of the surface motor are given in Table 1. The results of one phase in X-direction of the SRPM are adopted and the remaining phases of the motor will give the same results. Each phase of SRPM can step $4 \mathrm{~mm}$ in X or Y direction. It can note that the high overshoots and oscillations in step response of SRPM are removed when utilizing the Neural-PID controller as shown in Fig 11. While Fig 12 shows the ability of the motor to work at different load conditions when using the Neural-PID controller. The thrust force is generated in one phase of SRPM in the X-direction is shown in Fig 13. This figure shows that the thurst force decrease until reach zero when the motor step movement is completed, which equals $4 \mathrm{~mm}$ also it shows that $X_{\text {off }}$ will not exceed $3 \mathrm{~mm}$. The position-inductance characteristic is shown in Fig 14. This figure proves the inductance starts at a minimum value and reaches a maximum value when the motor movement is completed. Figs 15 and 16 show the XY movement of SRPM when the motor is traveled through a rectangular and triangle target, respectively. These figures show the validity of the surface motor to achieve accurate tracing to the desired positions.

Table 1. The parameters of the SRPM.

\begin{tabular}{ll}
\hline Parameters & values \\
\hline Pole width & $6 \mathrm{~mm}$ \\
Pole pitch & $12 \mathrm{~mm}$ \\
Phase separation & $10 \mathrm{~mm}$ \\
Winding length & $30 \mathrm{~mm}$ \\
Wind width & $25 \mathrm{~mm}$ \\
Air gap width & $0.5 \mathrm{~mm}$ \\
Phase resistance & $2.5 \Omega$ \\
Aligned inductance & $19.2 \mathrm{mH}$ \\
Unaligned inductance & $11.5 \mathrm{mH}$ \\
The mass of moving platform (m) & $1.8 \mathrm{Kg}$ \\
Friction constant (b) & $0.08 \mathrm{~N}^{*} \mathrm{~s}^{*} \mathrm{~m}^{-1}$
\end{tabular}




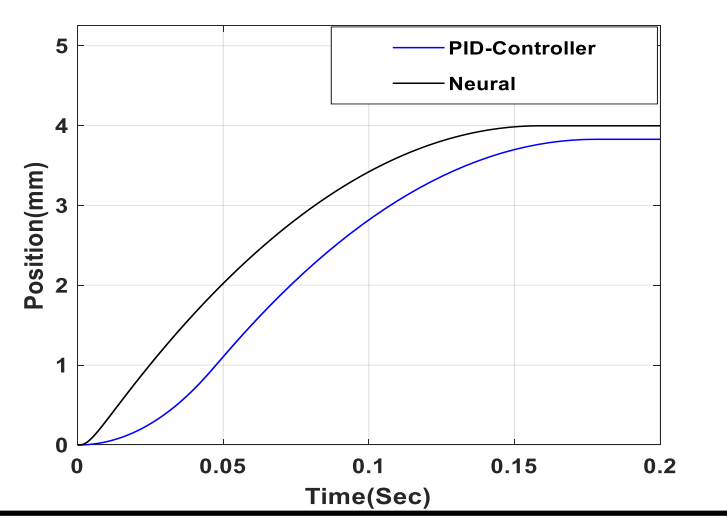

Fig 11. The Position response of one phase of SRPM in $\mathrm{X}$-direction with $\mathrm{m}=1.8 \mathrm{~kg}$.

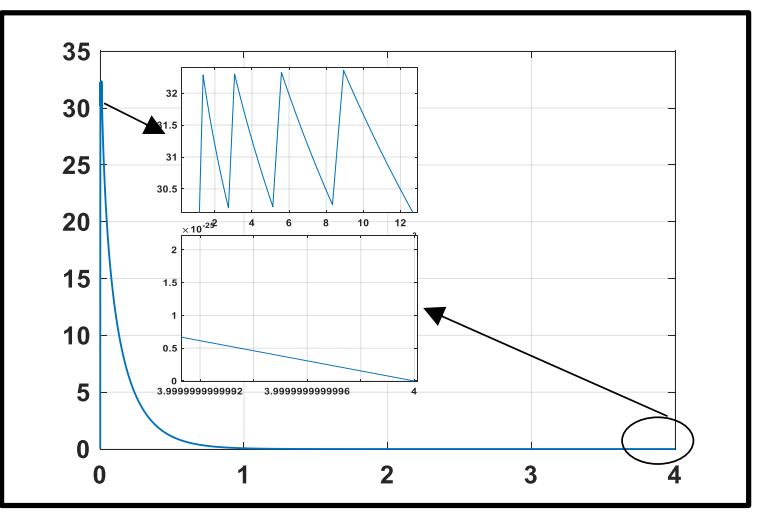

Fig 13. The force-position characteristic of one phase of SRPM in X-direction with Neural-PID controller at $\mathrm{m}=1.8 \mathrm{~kg}$.

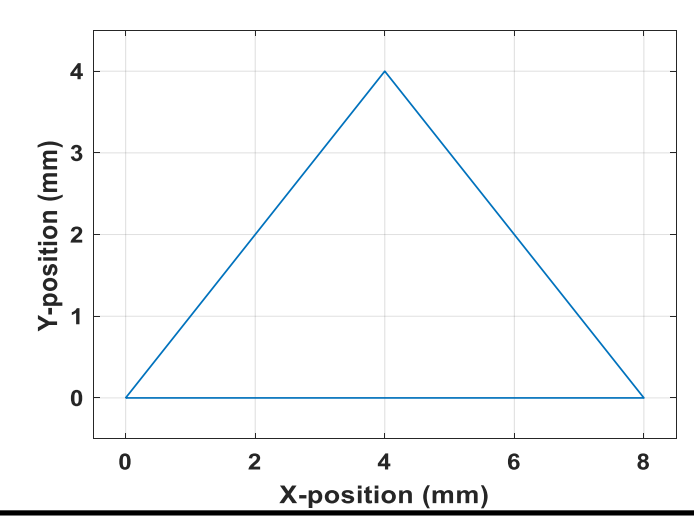

Fig 15. Square movement of the surface motor with Neural-PID controller.

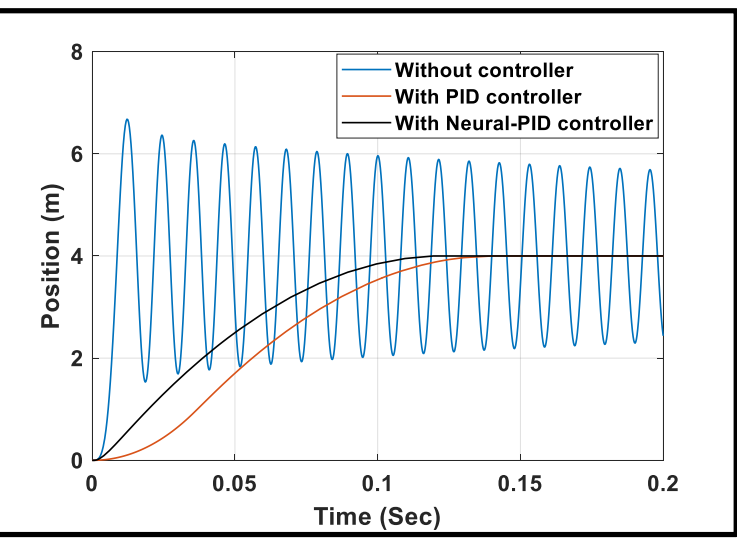

Fig 12. The Position response of one phase of SRPM in $\mathrm{X}$-direction with $\mathrm{m}=3 \mathrm{~kg}$.

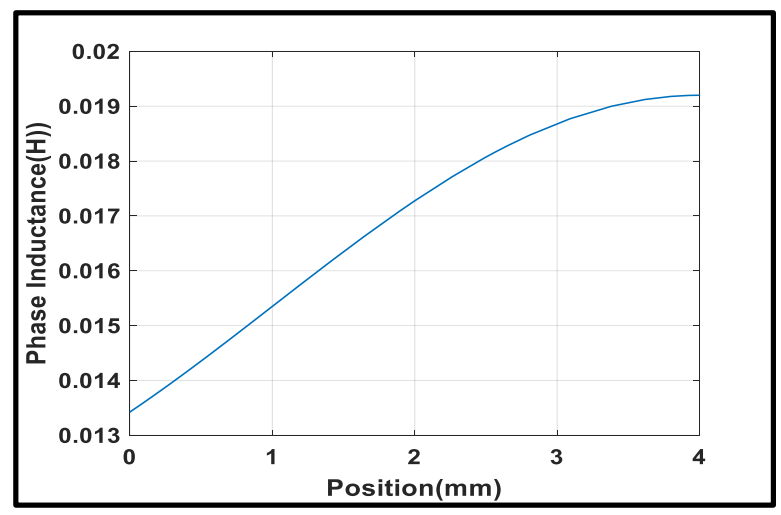

Fig 14. Phase inductance-position characteristics of the surface motor in $\mathrm{X}$-direction with Neural-PID controller at $\mathrm{m}=1.8 \mathrm{~kg}$.

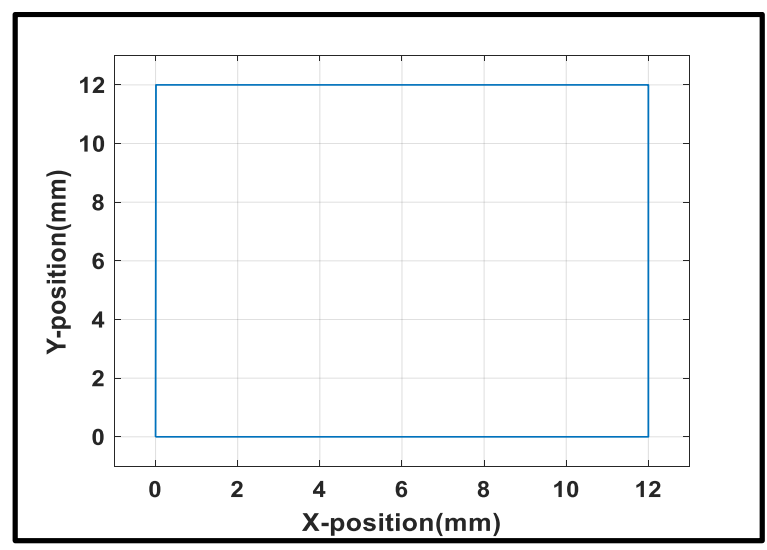

Fig 16. Triangle movement of the surface motor with Neural-PID controller.

\section{Conclusions}

The modeling of SRPM and the Neural-PID controller has been designed and tested using Matlab/Simulink software. The simulation results show that the control performance of the SRPM with the Neural-PID controller is 
better than the PID controller. The SRPM performance with Neural-PID controller has no oscillation, no overshoot, short rise time, strong robustness to load variation, and smooth and precise final position. The simulation results prove the proposed Neural-PID controller is effective for the SRPM to compare with the traditional PID controller. The main results from this work are concentrated on position regulation with neural technique. Therefore, it would be interesting to focus on velocity control using traditional controller (PID), fuzzy and neural strategies.

\section{References}

[1] L. Xuepeng, M. Xuesong, and W. Xutang, "Model and Control of Novel Surface-Motor in Plane Motion," Journal of the Korean Society for Industrial and Applied Mathematics, vol. 8, no. 2, pp. 39-49, 2004.

[2] L. Szabo, D.-C. Popa, V. Iancu, E. Kovacs, and F. Toth, "On the Usefulness of Simulation in Designing a Permanent Magnet Modular Surface Motor for Advanced Mechatronic Systems," In 2006 IEEE International Conference on Mechatronics, pp. 88-93: IEEE, July 2006.

[3] J. Pan, N. Cheung, W. Gan, and S. W. Zhao, "A novel planar switched reluctance motor for industrial applications, "IEEE Transactions on Magnetics, vol. 42, no. 10, pp. 2836-2839, October 2006.

[4] J. Pan, N. C. Cheung, and J. Yang, "High-precision position control of a novel planar switched reluctance motor," IEEE Transactions on Industrial and Electronics, vol.52, no.6, December 2005.

[5] J. F. Pan and N. C. Cheung, "An adaptive controller for the novel planar switched reluctance motor," IET Electric Power Applications, vol. 5, no. 9, pp. 677-683, November 2011.

[6] G. Z. Cao, J.-L. Fang, S.-D. Huang, J.-A. Duan, and J. F. Pan, "Optimization design of the planar switched reluctance motor on electromagnetic force ripple minimization," IEEE Transactions on Magnetics, vol. 50, no. 11, November 2014.

[7] H. J. Hu, G.-Z. Cao, S. D. Huang, Z.- Z. Xu, and C. Wu, "A DSP-based sliding mode controller for the planar switched reluctance motor," In 2017 IEEE International Electric Machines and Drives Conference (IEMDC), pp. 1-6, May 2017.

[8] Z.-Y. Hu, S.-D. Huang, G.-Z. Cao, L. Chen, G. Jing, and Y. Liu, "Predictive Position Control With Stable Control Parameters of Planar Switched Reluctance Motors," In 2020 10th Institute of Electrical and Electronics Engineers International Conference on Cyber Technology in Automation, Control, and Intelligent Systems (CYBER), pp. 318-321: IEEE, October 2020.

[9] J. M. Yang, Q. Zhong, N. C. Cheung, and S. W. Zhao, "On Control of Planar Switched Reluctance Motor," Proceedings of the $17^{\text {th }}$ World Congress, The International Federation of Automatic Control, Seoul, Korea, vol. 41, no. 2, pp. 1570215707 , July 2008.

[10] W. Zaafrane, M. Dursun, and H. Rehaoulia, "Double-sided linear switched reluctance motor analysis and modeling including end-effect," in 2018 5th International Conference on Electrical and Electronic Engineering (ICEEE), pp. 124128: IEEE, May 2018.

[11] S. Zhou and H. Lin, "Modeling and simulation of switched reluctance motor double closed-loop control system," Proceeding of the $6^{\text {th }}$ World Congress on Intelligent Control and Automation, Dalian, China, PP. 6151-6155, June 2006.

[12] R. S. Burns, “Advanced control engineering”, University of Plymouth, UK, pp.348-354, Elsevier,2001. 